\title{
Der Umgang mit Fehlern als Merkmal betrieblicher Fehlerkultur und Voraussetzung für Professional Learning
}

\section{Christian Harteis, Johannes Bauer und Helmut Heid}

Moderne Unternehmen folgen Philosophien, wonach Beschäftigte Kompetenzen entwickeln und ihr Unternehmen als lernende Organisation begreifen sollen. Das soll das Potenzial des Unternehmens steigern, auf schwer antizipierbare Anforderungen angemessen reagieren zu können. Fehler stellen in diesem Kontext spezielle Lerngelegenheiten dar, zumal flexible Strukturen die Wahrscheinlichkeit erhöhen, dass Fehler geschehen. Jedoch reicht alleine ein Bekenntnis zu einer positiven Feblerkultur nicht aus, damit im betrieblichen Arbeitsalltag aus Fehlern gelernt wird. Es bedarf eines Umgangs mit Fehlern, der Merkmale einer positiven Fehlerkultur erfüllt. Dieser Beitrag klärt Voraussetzungen des Lernens aus Fehlern und berichtet Ergebnisse von Studien mit Beschäftigten aus unterschiedlichen Industrie- und Dienstleistungsunternehmen.

In der erziehungswissenschaftlichen Debatte um Lernen in der beruflichen Tätigkeit lassen sich zwei Denkrichtungen identifizieren, die in jüngerer Zeit an Aufmerksamkeit gewonnen haben. Die eine Richtung setzt an der Ausgestaltung betrieblicher Arbeitsorganisation an und interpretiert den Arbeitsplatz der Beschäftigten als Lehr-Lern-Situation, die mehr oder minder günstige Voraussetzungen für die individuelle Kompetenzentwicklung der Beschäftigten aufweisen kann (z.B. Appelbaum \& Gallagher, 2000; Billett, 2001). Die andere Richtung setzt bei den Beschäftigten an und analysiert Merkmale und Einflussfaktoren individueller Lernprozesse von Beschäftigten (z.B. Eraut, 2002; Simons \& Ruijters, 2004). Im deutschsprachigen Raum wird von Lernen am Arbeitsplatz (Dehnbostel, 2003) gesprochen, im internationalen Kontext scheint sich der Begriff vom Professional Learning (Gruber, Harteis \& Rehrl, 2005) zu etablieren.

In jeder der angesprochenen Richtungen stellt der Umgang mit Fehlern ein pädagogisch relevantes Thema dar. Betriebe verfolgen nicht primär Lernzwecke, allerdings werden auch in Betrieben Fehler gemacht, die für Lernprozesse und Lerneffekte bedeutsam sind. Fehler sind Fehler und insofern unerwünscht, aber sie sind praktisch unvermeidbar. Deshalb kann es nicht nur um die Vermeidung von Fehlern gehen, es muss auch eine Strategie des Umgangs mit Fehlern ent- 
wickelt werden. Der erziehungswissenschaftliche Ansatz des Lernens aus Fehlern - wie er beispielsweise von Oser, Hascher und Spychiger (1999) für den schulischen Kontext entwickelt wurde - begünstigt eine Pädagogisierung von Fehlern, der Vertreter betrieblicher Praxis skeptisch gegenüberstehen.

In diesem Beitrag thematisieren wir ein theoretisches Rahmenmodell zur Fehlerkultur in Betrieben, das in einer exemplarischen Studie einer empirischen Überprüfung unterzogen wurde. Im Folgenden stellen wir den theoretischen Ansatz vor und berichten über empirische Befunde eines zweijährigen Forschungsprojektes, das u.a. den lernförderlichen Umgang mit Fehlern im Betrieb zum Gegenstand hatte. Weswegen dieser Umgang mit Fehlern in Betrieben überhaupt von Bedeutung ist, wird zu Beginn des Artikels dargelegt. Anschließend werden einige grundlegende Gedanken über die generelle Problematik des Fehlerthemas entwickelt, ehe das theoretische Rahmenmodell zur Fehlerkultur vorgestellt wird. Schließlich wird ein Überblick über die wichtigsten Befunde aus dem Forschungsprojekt gegeben, und es werden Schlussfolgerungen aufgezeigt.

\section{Die Bedeutung des Lernens aus Fehlern in Betrieben}

Moderne Konzepte betrieblicher Arbeitsorganisation rechnen mit Umweltbedingungen steten Wandels und zunehmenden Wettbewerbs. Als entscheidende Erfolgsmerkmale wettbewerbsfähiger Betriebe werden Flexibilität und die Fähigkeit, sich rasch auf wechselnde Kundenbedürfnisse einzustellen, angesehen (Picot, Reichwald \& Wigand, 2003). Kernansatz der seit den 1990er Jahren entwickelten Organisationskonzepte ist eine Dezentralisierung von Entscheidungsbefugnis und eine Deregulierung von betrieblichen Abläufen. Die Hierarchien wurden verflacht und betriebliche Kontrollinstanzen abgeschafft, damit die Beschäftigten in den ausführenden Tätigkeiten rasch auf neue Anforderungen reagieren können. In Folge der Delegation von Verantwortung (Heid, 2005) stiegen gleichzeitig die Anforderungen an die Beschäftigten: Den neuen Ansätzen folgend müssen die Beschäftigten nun eine Vielzahl von Handlungsalternativen (und deren Nebeneffekte) erkennen und bewerten, ehe sie eine Entscheidung treffen (Hitt, Black \& Porter, 2005). Sie folgen nicht mehr vorgegebenen Arbeitsschritten, sondern sie sind auf ihre individuelle Kompetenz angewiesen.

Je mehr die Beschäftigten jedoch selbst für die konkrete Ausgestaltung von Arbeitsprozessen und deren Veränderung zuständig sind, desto mehr steigt auch die Wahrscheinlichkeit, dass Fehler geschehen, mit denen wiederum im Arbeitsalltag umgegangen werden muss. Es gibt Ansätze, die Fehler in komplexen Organisationen als unvermeidbar erachten (Kühl, 2000). Um den Umgang mit Fehlern bewältigen zu können, benötigen die Beschäftigten ein höheres Maß individueller Kompetenz als dies in streng und zentral reglementierten Arbeitsorganisationen der Fall war. Dass in der Management-Literatur von Humankapi- 
tal und Humanressourcen gesprochen wird, zeigt die hohe Bedeutung, die der individuellen Kompetenz der Beschäftigten zugesprochen wird. Das neue Paradigma moderner Organisationskonzepte lässt sich folgendermaßen zusammenfassen (Appelbaum \& Gallagher, 2000):

1. Beschäftigte verfügen zu einem gewissen Grad über Autonomie und Kontrolle über die eigenen Arbeitsaufgaben und -methoden. D.h. die Arbeitsaufgaben sind so neustrukturiert, dass Beschäftigten Entscheidungen über den Arbeitsprozess übertragen werden.

2. Von den Beschäftigten wird erwartet, dass sie sich in Projektgruppen einbinden, in deren Rahmen Problemlösungen erarbeitet werden. Zudem sollen sie sich an der Verbesserung betriebsinterner Abläufe beteiligen, insbesondere sobald Fehler zu Problemen führen. D.h. Beschäftigte müssen über professionelle Expertise verfügen, damit sie Probleme identifizieren und Lösungen kommunizieren können.

3. Die Arbeit wird sowohl in selbstgesteuerten, in den Produktionsprozess integrierten Teams organisiert als auch in temporären, aus den regulären Abläufen herausgenommenen Projektgruppen kontrolliert.

4. Von den Beschäftigten wird erwartet, dass sie aus betrieblichen Vorkommnissen und insbesondere aus Fehlern lernen.

Aus diesem Paradigma ergibt sich die große Bedeutung, die einem lernförderlichen Umgang mit Fehlern sowie der Entwicklung einer lernförderlichen Fehlerkultur im Betrieb zukommt. Bei einer genaueren Betrachtung stellt sich aber heraus, dass ein grundlegendes Problem der Fehlerthematik darin besteht, zu klären, was genau ein Fehler ist.

\section{Fehler - eine theoretische Begriffsklärung}

Auf den ersten Blick erscheint die Thematisierung von Fehlern als eine Auseinandersetzung mit dem scheinbar klar strukturierten Sachverhalt, dass «etwas falsch gelaufen» sei. Gerade im Kontext sozialer Gebilde wie Betrieben ist die Festlegung darauf, was ein Fehler ist, ein kompliziertes, von impliziten Definitionsprozessen bestimmtes Unterfangen. Bei der Definition von Fehlern sind folgende Ebenen zu berücksichtigen:

1. Die inhaltliche Ebene: Was wird als Fehler bezeichnet?

2. Die normative Ebene: Weswegen wird etwas als Fehler bezeichnet?

3. Die personale Ebene: Wer bezeichnet etwas als Fehler?

4. Die aktionale Ebene: Was führte zum Fehler und welche Konsequenzen folgen daraus?

Eine kurze Erörterung dieser Ebenen soll die prinzipielle Problematik der Thematisierung von Fehlern in betrieblichen Kontexten verdeutlichen. 
Die inhaltliche Ebene: Was wird als Fehler bezeichnet? In einer allgemein gehaltenen Definition bezeichnen Oser, Hascher \& Spychiger (1999) Fehler als einen von einer oder mehreren Normen abweichenden Sachverhalt oder Prozess. Sie wählen damit eine Definition auf hohem Abstraktionsniveau, wie sie auch in internationalen Arbeiten gewählt wird (Cannon \& Edmondson, 2001; Nierenberg, 1996). Damit sind Fehler zum einen etwas, das von als normal geltenden oder als Norm anerkannten Gesichtspunkten abweicht. Diesem Aspekt soll auf der folgenden zweiten Ebene nachgegangen werden. Zum anderen können Fehler sowohl Sachverhalte als auch Prozesse betreffen. Wenn aus Fehlern gelernt werden soll, so scheint eine Klärung dessen unverzichtbar, woraus nun konkret gelernt werden soll. Läuft in einer Automobilfirma ein Fahrzeug in beschädigtem Zustand vom Band, so kann das defekte Fahrzeug selbst den Fehler «darstellen", es können Arbeitsschritte sein, die von einer Gütenorm abweichen, es können die falschen Schritte ausgeführt oder es können falsche Entscheidungen getroffen worden sein, die den einzelnen Arbeitsschritten zugrunde liegen. Allein dieser kurze Ausflug in betriebliche Realität verdeutlicht, dass die Frage der Fehlerdefinition keineswegs leicht zu klären ist. Denn egal, welche Fokussierung vorgenommen wird, sie stellt jeweils nur einen Teilausschnitt der Wirklichkeit in den Blickpunkt des Interesses, der das als wichtig Erachtete von dem als unwichtig Erachteten trennt. Diese Auswahl könnte interpersonal durchaus variieren.

Der Vorteil einer wie oben dargestellten allgemeinen Interpretation von Fehlern als Normabweichungen besteht darin, dass zunächst keine Unterscheidung und daher keine Abwägung unterschiedlicher Kategorien oder Qualitäten von Fehlern vorgenommen werden muss. Auf dieser Analyseebene spielt es (noch) keine Rolle, ob das Ziel verfehlt wurde, weil die Handlungspläne bzw. das Wissen unzureichend waren oder ob die Handlungen an sich unzureichend ausgefallen sind - um eine Unterscheidung von Reason (1990) aufzugreifen. Auch fallen hier Fragen der Unterscheidung verschiedener Fehlleistungen (Norman, 1989) noch nicht an.

Unberücksichtigt bleibt in dem bislang Geschilderten darüber hinaus die Tatsache, dass auch die Unterlassung einer Handlung Gegenstand einer Fehleranalyse sein kann (Reason, 2001). Bei diesem Aspekt wird sofort deutlich, dass ein Fehler keineswegs gegenständlicher Natur ist. Zwar kann ein Fehler quasi vergegenständlicht sein, aber ein Fehler ist stets eine wertende Zuschreibung zu eben einem Sachverhalt oder einem Vorgang. Dinge, (unterlassene) Handlungen, Entscheidungen sind keine Fehler, sondern sie werden als solche bezeichnet. Diese für die weitere Betrachtung immens bedeutsame Feststellung impliziert, dass die Fehlerkategorie eine prinzipiell diskussionswürdige und -bedürftige Einstufung eines Beobachtungsobjektes darstellt, die - damit wird zur zweiten Ebene übergeleitet - abhängig von Bewertungsmaßstäben ist. 


\section{Normative Ebene: Weswegen wird etwas als Fehler} bezeichnet?

Wenn etwas als Fehler bezeichnet wird, dann liegt gemäß der o.g. Definition eine Abweichung von etwas als normal Erachtetem oder als (Qualitäts-)Norm Gesetztem vor (Frese \& Zapf, 1991). Die Beschaffenheit eines Beobachtungsgegenstandes weicht von einer Norm ab. Fehler sind demnach eine Bewertungskategorie, die aus einer Relation zwischen einem beobachteten Gegenstand oder Vorgang und einer Norm(konstellation) resultiert.

So wie die Wahl des Beobachtungskriteriums eine Selektion aus der Gesamtheit der betrieblichen Realität darstellt, so stellt der Bezug auf eine oder mehrere Normen eine Selektion dar. Wenn Akteur und Beobachter unterschiedliche Personen sind, dann ist es möglich, dass Normen, an denen das Handeln ausgerichtet ist, nicht mit den Normen deckungsgleich sind, die zu einer Fehlerbewertung führen. Problematisch wäre dies zumindest in solchen Fällen, in denen diese beiden normativen Bezugssysteme unvereinbar sind.

Personale Ebene: Wer bezeichnet etwas als Fehler?

Insbesondere im betrieblichen Kontext ist nicht immer klar, welche Person die entscheidende Instanz zur Feststellung eines Fehlers ist. Da dort isoliertes Einzelhandeln nicht an der Tagesordnung ist und zudem (in den meisten Fällen) ein hierarchisches Gefälle in der Organisationsstruktur angelegt ist, wird es häufig der Fall sein, dass nicht die handelnde Person die Entscheidung über die Fehlerbewertung vornimmt, sondern dies von einer anderen Person vorgenommen wird.

In einem solchen Fall werden Fragen der Legitimation aufgeworfen, wie weit die urteilende Person kraft ihrer Kompetenz (sowohl im Sinne von Fähigkeit als auch von Zuständigkeit) zu einem (akzeptierten) Urteil gelangen kann. Mit der Legitimationsfrage verbunden ist der Gesichtspunkt der Kriterientransparenz. Wenn eine außen stehende Person das Handeln einer anderen Person als fehlerhaft bewertet, kann dies aufgrund transparenter wie auch intransparenter Bewertungsmaßstäbe geschehen. Selbst transparente Kriterien können bei wenig präziser Ausformulierung Deutungsspielraum eröffnen und die definitionsschwächere Person benachteiligen.

\section{Aktionale Ebene: Was führt zum Fehler und welche Konsequenzen zieht er nach sich?}

Auf dieser Ebene sind zwei Gesichtspunkte für die Fehlerbeschreibung von Bedeutung, nämlich zum einen die Frage, wodurch ein Fehler zustande kommt und zum anderen die Frage, welche Folgen aus dem Fehler erwachsen. Wenngleich diese Ebene getrennt von den anderen gesteigerte Bedeutung für die Bewertung von Fehlern haben sollte, so ist sie doch auch in Zusammenhang mit der Definition von Fehlern zu sehen.

Der handlungslogisch erste Aspekt der Antezedenzen von Fehlern ist mit Fragen verknüpft, wie weit die handelnde Person Kontrolle über die Handlungssi- 
tuation hatte, ob auf der sensumotorischen der perzeptiv-begrifflichen oder der intellektuellen Regulationsebene (Hacker, 1998) Fehler geschehen, ob der Fehler vorhersehbar und ob er vermeidbar war. So ist es eine Frage individueller Wertung, ob von einem Fehler gesprochen werden soll, wenn die handelnde Person gar nicht über das Wissen verfügte, das unentbehrlich ist, um die Konsequenz des Handelns einzuschätzen zu können, oder wenn sie in der Situation gar unzurechnungsfähig war. Für die Frage, ob ein Fehler vorhersehbar war, ist also der Bestand individuellen deklarativen und prozeduralen Wissens entscheidend (Zapf, Frese \& Brodbeck, 1999), und für die Frage, ob er vermeidbar war, ist das Vorhandensein realisierbar erscheinender Handlungsalternativen von Bedeutung (Heid, 1991).

\section{Merkmale einer Lernförderlichen Fehlerkultur}

Der Begriff der Fehlerkultur bezieht sich einerseits auf die Bedeutung, die Fehlern in der täglichen Arbeitspraxis zugesprochen wird, und andererseits auf die Konsequenzen, die sich nach aufgetretenen Fehlern einstellen (Remdisch, 2002). In diesem Abschnitt soll auf diese beiden Aspekte unter der Perspektive theoretischer Modelle des Lernens aus Fehlern eingegangen werden, um Merkmale einer lernförderlichen Fehlerkultur aufzuzeigen.

Die Bedeutung von Fehlern kann prinzipiell auf einem Kontinuum zwischen zwei Extrempositionen beschrieben werden (Bauer, 2004): In der einen Extremposition werden Fehler als Übel und als möglichst zu vermeidende Vorfälle betrachtet. Diese Vorstellung findet sich in zahlreichen Ansätzen betrieblicher Arbeitsorganisation, insbesondere bei denen eine Null-Fehler-Strategie ausgegeben (Hummel \& Malorny, 2002) und ein «reibungsloser Verlauf in allen Unternehmensbereichen» (Münchrath \& Runge, 2000, S.102) gefordert wird. Die andere Extremposition besteht darin, Fehler als nützliche, nutzbare, zumindest als prinzipiell unvermeidbare Ereignisse anzusehen, die wichtige Informationen zur Verbesserung des bisherigen Vorgehens beinhalten. Derlei Vorstellungen stehen in der Tradition systemischer Denkansätze (Kühl, 2000).

Gegen die Utopie einer Null-Fehler-Strategie spricht die Tatsache, dass Entscheidungen in Betrieben in der Regel in komplexen Zusammenhängen - und somit unter unvollständiger Information und nicht völliger Transparenz möglicher Auswirkungen - zu treffen sind. Dies spricht für die prinzipielle Fehleranfälligkeit betrieblicher Entscheidungen. Unter dieser Voraussetzung erscheint eine Strategie der Fehlervermeidung nicht unproblematisch:

- Eine durch bürokratische und technische Strukturen zu gewährleistende Vermeidung von Fehlern versagt und zeitigt umso schlimmere Folgen, je mehr die Akteure sich in vermeintlicher Sicherheit wiegen und nicht mit dem Auftreten von Fehlern rechnen; stellvertretend für alle anderen Fälle steht hier das Unglück in Tschernobyl (Dörner, 2003). 
- Wird in Betrieben eine ablehnende Haltung gegenüber Fehlern praktiziert, so führen auftretende Fehler wahrscheinlich zu Appellation von Verantwortung, Schuld und Sanktion (Rybowiak, Garst, Frese \& Batinic, 1999). Das begünstigt die Reaktion, dass Betroffene versuchen, Fehler zu verheimlichen. Damit bestünde keine Möglichkeit mehr für Dritte, aus einem solchen Vorfall zu lernen.

Eine lernförderliche Fehlerkultur äußert sich darin, dass Fehler nicht im unbegründeten Sicherheitsgefühl aus dem Fokus der Aufmerksamkeit ausgeblendet werden und dass sie nicht zu Schuldzuweisungen und negativen Konsequenzen für die Akteure führen. Ein aktuelles Beispiel professioneller Akteure findet sich im Mannschaftssport. Der Trainer der Deutschen Fußball-Nationalmannschaft hat die Spiele im Vorfeld der anstehenden Weltmeisterschaft zu Lerngelegenheiten erklärt. Seine Spieler hätten die Testbegegnungen als Ausgangspunkt für ihre individuelle Weiterentwicklung zu begreifen. Fehler seien erlaubt, da ohnehin unvermeidbar und angesichts jugendlicher Unerfahrenheit in der Mannschaft auch zwangsläufig. Freilich ist es nicht Ziel von Unternehmen, Fehler zu begehen. Wenn man jedoch die Auffassung akzeptiert, dass sie sich nicht vermeiden lassen, dann gewinnen sie im betrieblichen Alltag die Bedeutung von informativen Ereignissen, die es zu analysieren und auf dieser Basis systematisch und wirksam zu «beherrschen» gilt. Im Vordergrund steht dann die Frage, wie auf Fehler zu reagieren ist und nicht die Frage, wer für die Folgen zu haften hat. Stellungsfehler im Abwehrverhalten der deutschen Mannschaft dienen den Spielern als Information über eine angemessene Verhaltensänderung, in Produktionsbetrieben ist ein Maschinenstillstand ohne Fehleranalyse ein schicksalhaftes Ereignis, aber ein Maschinenstillstand bietet bei gründlicher Ursachenforschung reichhaltige Informationen zur Vermeidung weiterer Stillstände.

\section{Lernen aus Fehlern}

Obgleich das Lernen aus Fehlern in verschiedenen lernpsychologischen Ansätzen impliziert wird (z.B. Lernen durch Versuch und Irrtum oder Problemlösen) erfährt es in den Ansätzen und Forschungsarbeiten aus der Pädagogischen Psychologie nur wenig Beachtung (Oser, Hascher \& Spychiger, 1999). Zentrale Voraussetzungen des Lernens aus Fehlern sind nach Oser \& Spychiger (2005) ein Unterbruch, Betroffenheit und Reflexion, die in der Theorie negativen Wissens zu einem Lernen aus Fehlern führen. Mit Unterbruch ist ein Innehalten der Tätigkeit gemeint, das der kognitiven Erfassung einer Normabweichung Raum gibt. Betroffenheit führt dazu, dass die handelnde Person sich als Ursache der Normabweichung begreift, um schließlich in Reflexion über die Ursachen des Fehlers und mögliche Handlungsalternativen einzutreten. Dadurch wird Wissen aufgebaut, wie und warum etwas nicht funktioniert wie es besser gemacht werden kann. 
Dieser theoretische Ansatz setzt voraus, dass die Bewertungskriterien, die zur Fehlerklassifizierung führen, klar sind. Unklar ist, ob dies in betrieblichen Zusammenhängen - beispielsweise wenn Vorgesetzte Fehler bei ihren Mitarbeitern aufdecken - regelmäßig der Fall ist. Ein Verständnis für die Ursache eines Fehlers zu entwickeln, setzt die Bestimmung, Geltung und Anwendung eines Bewertungskriteriums voraus. Deswegen erscheint Lernen aus Fehlern im betrieblichen Kontext nur möglich, wenn die Bewertungskriterien bekannt und präzise operationalisiert sind. Eine weiterführende Frage ist die nach der Mitwirkung Beschäftigter an der Kriterienbestimmung: Wie weit können Beschäftigte an der Definition von Bewertungskriterien partizipieren? Zumindest aus motivationstheoretischer Perspektive erscheinen Lernprozesse wahrscheinlicher, wenn diejenigen, die aus Fehlern lernen sollen, Mitsprache bei der Definition von Fehlern besitzen.

Eine weitere Voraussetzung, die der Ansatz der Osergruppe impliziert, besteht darin, dass die handelnde Person Rückmeldung über die Normabweichung erhält. Gerade in komplexen Systemen ist der Effekt des eigenen Handelns möglicherweise nicht unmittelbar zu erkennen, weswegen das Feedback an die Beschäftigten dann erfolgen muss, wenn diese keine Gelegenheit haben, ihren Fehler selbst zu erkennen (etwa wenn sie in einer späteren Phase des Produktionsprozesses auftreten).

Im Umgang mit Fehlern fördert eine Kultur konstruktiver, wertschätzender und angstfreier Zusammenarbeit das Lernen aus Fehlern. Dabei werden in der internationalen Literatur Aspekte des Fehlermanagements betont, das einen kooperativen und lösungsorientierten Ansatz im Gegensatz zu einem schuldzuweisungsorientierten fordert (Tjosvold, Yu \& Hui, 2004; Zapf, Frese \& Brodbeck, 1999). Der Aspekt der Straffreiheit wird insbesondere im Ansatz des Critical Incident Reporting thematisiert, das auf ein umfassendes Wissensmanagement von Fehlern und Beinahe-Fehlern abzielt (Hofinger, 2002). Spychiger, Oser, Hascher \& Mahler (1999) fanden im Kontext des Schulbetriebs drei für Lernprozesse einflussreiche Kategorien des Umgangs mit Fehlern: (a) Reaktionen der Lehrperson (im Betrieb eventuell analog: des Vorgesetzten), (b) die Selbsteinschätzung im Umgang mit Fehlern sowie (c) der emotionale Umgang mit Fehlern. Vor allem die Reaktion Anderer auf Fehler dürften in einem Zusammenhang mit der Emotionslage bei Personen stehen, die Fehler begehen.

Der theoretische Rahmen an Voraussetzungen für Lernen aus Fehlern lässt sich demzufolge in vier Kriterien eines lernförderlichen Umgangs mit Fehlern zusammenfassen:

1. Es muss klar sein und Übereinstimmung zwischen allen Beteiligten herrschen, was als Fehler bezeichnet wird.

2. Es muss klar sein und Übereinstimmung herrschen, weswegen etwas als Fehler bezeichnet wird. Dabei wirkt Partizipation an der Festlegung der Bewertungskriterien positiv auf die Motivation, aus Fehlern zu lernen. 
3. Es bedarf der Fehleranalyse und des Feedbacks an die handelnde Person.

4. Es muss eine positive Fehlerkultur vorherrschen.

\section{Der Umgang mit Fehlern im Betrieb - ein empirischer Zugang}

Die empirische Befundlage zum Umgang mit Fehlern im Betrieb ist aus verschiedenen Gründen unbefriedigend. Zum einen gestaltet sich der Feldzugang schwierig, da Betriebe der Veröffentlichung von Fehlern - und das geschieht beim Forschungszugang - nicht Vorschub leisten wollen. Zum anderen kann ein empirischer Zugang allenfalls exemplarisch realisiert werden. Nicht nur, weil die Ziehung von Repräsentativstichproben kaum realisierbar erscheint, sondern auch, weil der Umgang mit Fehlern nur im Zusammenhang mit einem konkreten Fallbeispiel sinnvoll ist.

In den beiden vorhergehenden Abschnitten wurde das theoretische Rahmenmodell für eine Fehlerkultur in Betrieben skizziert und in vier Kriterien eines lernförderlichen Umgangs mit Fehlern zusammengefasst. In diesem Abschnitt soll nun die erste empirische Überprüfung dieses Modells in der betrieblichen Praxis dargestellt werden, indem auf empirische Befunde aus einem durch die Deutsche Forschungsgemeinschaft geförderten Projekt (Az He1158/4-2) eingegangen wird, die an anderer Stelle im Detail publiziert sind (Bauer, Festner, Harteis, Heid \& Gruber, 2004; Harteis, Bauer, Festner, Gruber \& Heid, 2005). Für die Untersuchungen, in denen das Problem des lernförderlichen Umgangs mit Fehlern im Betrieb aufgeworfen wurde, konnten Beschäftigte aus unterschiedlichen deutschen Großunternehmen - Automobilhersteller, Elektronikproduzenten, Dienstleister - gewonnen werden, wobei betriebsspezifische Analysen nicht vorgesehen waren (eine explizite Nennung der Unternehmen unterbleibt hier aufgrund der sensiblen Thematik). Die Verteilung der Stichproben über diverse Betriebe hinweg sollte jedoch einen unternehmensspezifischen Bias vermeiden helfen. Dieser Beitrag bezieht sich auf Erhebungen mit zwei verschiedenen Stichproben: An den quantitativen Erhebungen nahmen 160 Personen teil, von denen 52 aus dem Kreis von Führungskräften stammten und 108 keinerlei Führungsfunktion ausüben. Aus diesem Personenkreis wurden 28 Personen (jeweils 14 Personen aus beiden Teilgruppen) ausgewählt, um mit ihnen zusätzlich Telefoninterviews durchzuführen.

\section{Kriterium 1: übereinstimmung in der Definition von Fehlern}

Die Übereinstimmung in der Definition von Fehlern lässt sich durch Vorgabe von Beispielfällen ermitteln, indem Versuchspersonen darüber entscheiden sollen, ob sie die fiktiven Vorfälle in ihrem Unternehmen als Fehler bezeichnen würden. Die Fälle unterscheiden sich hinsichtlich der Reichweite ihrer direkten 
Auswirkung. Die scharfe Kontrastierung eindeutiger und weniger eindeutiger Beispielfälle war forschungsmethodologisch beabsichtigt, um die Diskrepanz zwischen sachlicher Analyse und Alltagskonvention aufzuzeigen. Tabelle 1 zeigt die Vorgaben und den Anteil der Antworten, wonach die Vorgaben als Fehler bezeichnet würden oder nicht.

\section{Tabelle 1: Ergebnisse zur Frage, was im Arbeitsumfeld der Versuchspersonen als} Fehler bezeichnet wird.

\begin{tabular}{|l|c|c|}
\hline Bei uns im Betrieb nennt man es einen Fehler, ... & ja/nein & Quote \\
\hline$\ldots$ wenn im Bereich der Produktion etwas schief geht. & $25 / 3$ & $89,3 \%$ \\
\hline$\ldots$ wenn im Bereich der Beziehungen zu Kunden etwas schief geht. & $22 / 6$ & $78,6 \%$ \\
\hline$\ldots$ wenn die Arbeit so organisiert ist, dass nicht alle Leute ausgelastet sind. & $10 / 16$ & $38,5 \%$ \\
\hline $\begin{array}{l}\ldots \text { wenn die Arbeit so organisiert ist, dass die Qualität der Arbeitsschritte } \\
\text { beeinträchtigt wird. }\end{array}$ & $23 / 3$ & $88,5 \%$ \\
\hline $\begin{array}{l}\ldots \text { wenn ein Betrieb so organisiert ist, dass die Interessen der Beschäftigten } \\
\text { vernachlässigt werden oder nicht zur Geltung gelangen. }\end{array}$ & $12 / 15$ & $44,4 \%$ \\
\hline$\ldots$ wenn der Betrieb die Fähigkeiten der Beschäftigten unzureichend nutzt. & $15 / 13$ & $53,6 \%$ \\
\hline $\begin{array}{l}\ldots \text { wenn die Beschäftigten im Betrieb nicht ausreichend unterstützt wer- } \\
\text { den, ihre Kompetenzen und Fähigkeiten weiter zu entwickeln. }\end{array}$ & $18 / 9$ & $66,7 \%$ \\
\hline $\begin{array}{l}\ldots \text { wenn die Arbeit so organisiert ist, dass die Gesundheit der Beschäftig- } \\
\text { ten gefährdet ist. }\end{array}$ & $26 / 2$ & $92,9 \%$ \\
\hline $\begin{array}{l}\ldots \text { wenn die Beziehungen zwischen Führungskräften und Mitarbeitern } \\
\text { bzw. Mitarbeiterinnen beeinträchtigt sind. }\end{array}$ & $18 / 9$ & $66,7 \%$ \\
\hline $\begin{array}{l}\ldots \text { wenn die Arbeitsbeziehungen zwischen Kolleginnen bzw. Kolleginnen } \\
\text { beeinträchtigt sind. }\end{array}$ & $15 / 13$ & $53,6 \%$ \\
\hline
\end{tabular}

Anmerkung: Die Zahlen weisen jeweils Zustimmung/Ablehnung zu den Aussagen aus. Quote $=$ Prozent der Ja-Antworten.

Wie aus den Daten hervorgeht, ist die Übereinstimmung in den Antworten unterschiedlich hoch. Während die gesundheitliche Beeinträchtigung der Beschäftigten oder die Beeinträchtigung von Produktionsprozessen und Arbeitsschritten von etwa 90 Prozent der Versuchspersonen als Fehler bezeichnet werden - und man daher von einer hohen Übereinstimmung sprechen kann -, sinken die Quoten, wenn beispielsweise Möglichkeiten der Kompetenzentwikklung oder die Beeinträchtigung von Arbeitsbeziehungen angesprochen werden.

Es ist somit offenbar nicht a priori davon auszugehen, dass in betrieblichen Kontexten alle Beteiligten darin übereinstimmen, ob ein Vorfall als Fehler zu bezeichnen ist. Vielmehr scheint man eine hohe Übereinstimmungsquote nur in eng begrenzten Bereichen erwarten zu können. Das hat jedoch Konsequenzen für die Gelegenheit, aus diesen Vorfällen oder Fehlern zu lernen. Solange Vorgänge nicht als Abweichung von einem Soll-Zustand wahrgenommen werden, solange ergibt sich keine Notwendigkeit für eine Änderung. 


\section{Kriterium 2: übereinstimmung in den Bewertungskriterien und Partizipation an deren Festlegung}

Eine Übereinstimmung in den Kriterien zur Bewertung eines Vorfalls lässt sich nur dann nachweisen, wenn verschiedene Personen auf den gleichen konkreten Vorfall Bezug nehmen. Das war in den durchgeführten Telefoninterviews nicht der Fall, weswegen zu diesem Punkt keine empirischen Befunde erzielt wurden.

Im Verlauf der Interviews sollten die Versuchspersonen einen gravierenden Fehler aus ihrem Arbeitskontext berichten. Hierbei nahmen sie großenteils auf Fehler Bezug, die ihnen selbst unterlaufen sind. In keinem dieser Fälle wurde berichtet, dass Unklarheit oder Uneinigkeit über die Bewertungskriterien bestand. Daraus kann jedoch nicht geschlossen werden, dass prinzipiell Übereinstimmung in den Bewertungskriterien besteht. Vielmehr werden offenbar vorzugsweise (für den Urteilenden) «klare» Fehler berichtet, wenn nicht explizit nach Uneinigkeiten gefragt wird.

Mit den Versuchspersonen wurden Kriterien für die Bewertung von Fehlern diskutiert und dabei in zwei Kategorien unterschieden. Die erste Kategorie bildeten Kriterien, die sich objektiv nachvollziehbar aus "harten» Fakten - wie etwa Messwerten - ergeben. Die zweite Kategorie bestand aus Kriterien, die sich eher aus vagen Wertungen ergeben. Für beide Kategorien wurden die Versuchspersonen befragt, ob sie in ihrem Arbeitsumfeld Einfluss auf die Festlegung harter und weicher Kriterien für die Bewertung von Fehlern nehmen könnten. Lediglich acht der 28 Versuchspersonen gaben an, keinerlei Einfluss auf die harten Kriterien zu besitzen, im Fall der weichen Kriterien waren es fünf Versuchspersonen. Die Gruppe der Befragten bestand zu gleichen Teilen aus Vorgesetzten und Mitgliedern der Belegschaft, eine statistische Überprüfung der Unterschiede zwischen beiden Gruppen zeigte jedoch kein signifikantes Ergebnis (Fisher-YatesTest, exakte Testung, zweiseitig: harte Kriterien $p<.68$; weiche Kriterien $p<.32$ ).

Aus motivationaler Perspektive ist der Eindruck der meisten Versuchspersonen, sie könnten auf die Festlegung der Kriterien zur Fehlerbewertung Einfluss ausüben, positiv zu werten. Zudem wurden keine Unstimmigkeiten in Bezug auf die Kriterien zur Bewertung von Fehlern berichtet. Allerdings können daraus keinerlei weiteren Rückschlüsse gezogen werden.

\section{Kriterium 3: Fehleranalyse und Feedback}

Die Analyse eines Fehlers wird als Voraussetzung dafür betrachtet, aus dem Fehler lernen zu können. Die Notwendigkeit des Feedbacks an die handelnde Person wurde für betriebliche Kontexte dadurch begründet, dass der Fehler möglicherweise gar nicht von der handelnden Person selbst aufgedeckt wird.

Im Zuge der Interviews wurde bei der Besprechung der Fehlerbeispiele die Art und Weise der Korrektur des Fehlers thematisiert. Die Versuchspersonen wurden gefragt, ob der Fehler beseitigt wurde, ob er dokumentiert und somit Dritten zugänglich gemacht wurde, ob Maßnahmen ergriffen wurden, den Feh- 
ler in Zukunft zu vermeiden und ob die handelnde Person Gelegenheit hatte, ihre Sicht der Dinge darzustellen. Diese Punkte können als Indizien für eine eingehende Fehleranalyse angesehen werden. Tabelle 2 fasst die Antworten zusammen:

Tabelle 2: Ergebnisse zur Frage, wie mit Fehlern umgegangen wird.

\begin{tabular}{|l|c|}
\hline & ja/nein \\
\hline Wurde etwas unternommen, um den Fehler zu beseitigen? & $19 / 2$ \\
\hline Wurde etwas unternommen, um den Fehler zukünftig zu vermeiden? & $19 / 5$ \\
\hline Wurde der Fehler dokumentiert oder anderen zugänglich gemacht? & $16 / 7$ \\
\hline Hatte die betreffende Person Gelegenheit, ihre Sicht der Dinge darzustellen? & $22 / 1$ \\
\hline
\end{tabular}

Anmerkung: Die Zahlen weisen jeweils Zustimmung (ja) /Ablehnung (nein) zu den Aussagen aus.

Die Fragen wurden von den meisten Versuchspersonen bejaht, statistisch relevante Unterschiede zwischen Führungskräften und Belegschaft ließen sich nicht nachweisen (Fisher-Yates-Test, exakte Testung, zweiseitig: $p$ zwischen .67 und 1). Insofern scheinen die von den Befragten geschilderten Fehler analysiert worden zu sein, mit einer Ausnahme auch mit der betreffenden Person. Hiermit ist auch die Frage des Feedbacks an die handelnde Person geklärt.

In Hinblick auf Fehleranalyse und Feedback scheinen somit günstige Voraussetzungen für ein Lernen aus Fehlern gegeben. Es stellt sich zwar auch hier die Frage, wie weit die Aussagen auf andere Fälle übertragen werden dürfen, aber es wäre bemerkenswert, würden die Versuchspersonen bei der Schilderung ihrer frei gewählten Beispiele jene bevorzugen, die analysiert und rückgemeldet wurden.

\section{Kriterium 4: Positive Fehlerkultur}

Fehlerkultur wurde in die beiden Dimensionen nach dem Stellenwert von Fehlern und dem Umgang mit Fehlern differenziert. Zur Ermittlung des Stellenwerts von Fehlern wurde der "Error Orientation Questionnaire» (Rybowiak et al., 1999) verwendet, der die individuelle Einschätzung verschiedener Merkmale von Fehlerorientierung misst. Faktorenanalytisch konnten drei Skalen extrahiert werden:

- Bewertung von Fehlern (9 Items, z.B. «ich mache lieber etwas falsch, als dass ich überhaupt nichts tue», Cronbachs $\alpha=.80$ )

- Strategien zum lernförderlichen Umgang mit Fehlern (5 Items, z.B. "wenn ich einen Fehler selbst nicht beheben kann, wende ich mich an meine Kolleg/inn/en», Cronbachs $\alpha=.75$ )

- Emotionen im Umgang mit Fehlern (6 Items, z.B. «ich schäme mich, wenn ich einen Fehler mache», Cronbachs $\alpha=.68$ ) 
Es wurden die Annahmen überprüft, ob Führungskräfte und Belegschaft sich in Teilaspekten der Fehlerorientierung unterscheiden würden. Die Befunde zeigten, dass sich bei der Bewertung von Fehlern ein zwar statistisch signifikanter (multivariate Varianzanalyse: $F(1.156)=6.1 ; p<.05)$, aufgrund geringer Effektstärke $(d=0.4)$ jedoch nicht bedeutsamer Unterschied zwischen beiden Gruppen feststellen lässt. Die Führungskräfte tendierten zu einer leicht positiveren Bewertung von Fehlern als die Belegschaft. Ein solcher Unterschied ließ sich jedoch bei den Strategien zum lernförderlichen Umgang mit Fehlern $(F<1)$ und bei den Emotionen im Umgang mit Fehlern mit $F(1.156)=1.7$ nicht in statistisch signifikantem Ausmaß nachweisen.

Günstige Bedingungen für Lernen aus Fehlern stellen diese Befunde insofern dar, als eine lernförderliche Bewertung von und ein ertragreicher Umgang mit Fehlern üblicherweise Führungskräften zugesprochen wird, in deren Aufgabenbereich seit jeher auch die Reflexion über innerbetriebliche Abläufe fällt. Dass sich hier kein Unterschied zwischen Führungskräften und Belegschaft nachweisen lassen, kann ein Indiz dafür sein, dass in diesem Punkt nun auch die Belegschaft «aufgeholt» hat und ein entsprechendes Verständnis von Fehlern aufbringt.

Bezüglich des Umgangs mit Fehlern wurde in den Telefoninterviews die Frage gestellt, wie die Versuchspersonen den Umgang mit den von ihnen geschilderten Beispielen in kurzen Worten charakterisieren würden. Die Antworten sind in Tabelle 3 zusammengefasst.

\section{Tabelle 3: Charakterisierung des Umgangs mit den geschilderten Beispielen.}

\begin{tabular}{|l|c|}
\hline & Häufigkeit \\
\hline Es wurden Gespräche geführt. & 12 \\
\hline Es erfolgte eine konstruktive Aufarbeitung des Falles. & 10 \\
\hline Es wurden den Betroffenen Handlungsalternativen aufgezeigt. & 4 \\
\hline Es erfolgte in erster Linie eine klare Schuldzuweisung. & 2 \\
\hline Es wurde gemeinsam versucht den Fehler zu vertuschen. & 1 \\
\hline Es wurden externe Gutachter bestellt. & 1 \\
\hline Der Fehler wurde einfach ignoriert. & 1 \\
\hline Es erfolgte Strafanzeige und fristlose Kündigung. & 1 \\
\hline Es erfolgte ein Wutausbruch der Führungskraft. & 1 \\
\hline
\end{tabular}

Aus diesen Antworten geht hervor, dass es zwar durchaus zu einem Umgang mit Fehlern kommt, der emotionale und motivationale Konsequenzen zeitigt, die das Lernen aus Fehlern eher beeinträchtigen als fördern (z.B. Wutausbruch, Schuldzuweisung). Sie zeigen aber auch, dass eine klare Mehrheit der Antworten auf einen konstruktiven Umgang mit den geschilderten Fehlern hinweist. 
Es bleibt jedem individuellen Standpunkt überlassen, diese Indizien einer lernförderlichen Fehlerkultur als erwartet und unerwartet anzusehen. Die Daten weisen sicher einen Bias dahingehend auf, dass die Teilnahme an der Fragebogenerhebung und an den Interviews freiwillig geschah und somit ein Feld erschlossen wurde, das für das Thema Lernen aus Fehlern aufgeschlossen war. Die Befunde stellen dennoch auch einen ersten empirischen Feldzugang in Betrieben dar, der zumindest verdeutlicht, dass weitere Untersuchungen in Betrieben aussichtsreich sind.

\section{Schlussfolgerungen}

Lernen aus Fehlern ist in mehrfacher Hinsicht ein relevantes, aktuelles Thema:

In der aktuellen erziehungswissenschaftlichen Literatur werden unter dem Schlagwort Professional Learning individuelle Lernprozesse am Arbeitsplatz thematisiert, wobei vor allem informelle, erfahrungsgebundene Prozesse hervorgehoben werden. Lernen aus Fehlern ist dem in besonderer Weise zuzuordnen, da es prinzipiell im Kontext von Vorgängen außerhalb formeller Lehr-Lern-Settings diskutiert wird.

Moderne Ansätze betrieblicher Arbeitsorganisation proklamieren dezentralisierte Entscheidungsprozesse und deregulierte Abläufe vor dem Hintergrund permanenten Wandels. Entscheidungen werden also stets unter Unsicherheit getroffen und können sich daher leicht als fehlerhaft erweisen. Um Fehler nicht zu wiederholen, ist Lernen aus Fehlern notwendig.

Die dargestellten Befunde erlauben Schlussfolgerungen für unterschiedliche Akteure in Betrieben. Hier soll auf die Unternehmensleitung, die Beschäftigten und die Bildungsverantwortlichen in Betrieben eingegangen werden.

Aus Sicht einer Unternehmensleitung stellen sich die Befunde einerseits positiv dar, denn sie deuten auf eine konstruktive Art der Fehleraufarbeitung hin. Führungskräfte und Belegschaft nehmen gleichermaßen günstige Voraussetzungen für das Lernen aus Fehlern wahr. Damit sind zentrale Ideen kompetenzfördernder Arbeitsorganisation (Harteis, 2002) erfüllt. Andererseits birgt die große Varianz von Fehlerinterpretationen jedoch die Gefahr, dass zahlreiche Vorgänge gar nicht als fehlerhaft wahrgenommen werden, obwohl dies aus distanzierter Perspektive angemessen wäre. In diesem Fall bestünde das Problem darin, dass Fehlentwicklungen unbeachtet und unkorrigiert bleiben. Denn es erscheint fragwürdig, ob Vorfälle, die nicht als Fehler gesehen werden, den Akteuren Anlass zur Reflexion und Ursachenforschung - und somit Lerngelegenheit - geben. Die Befunde zeigen jedenfalls zweierlei: Man kann weder davon ausgehen, dass vermeintlich offensichtliche Fehler als solche erkannt und gesehen werden. Noch kann man davon ausgehen, dass eine Unternehmensleitung ein klares Bild vor Augen hat, was in ihrem Betrieb von den Beschäftigten als Fehler angesehen wird. Gebauer, Groth \& Simon (2004) sehen «Paradoxiemanagement» als die 
Etablierung der Aufmerksamkeit für Fälle des Scheiterns bei gleichzeitiger Stabilität der Funktionserfüllung als zentrale Managementaufgabe.

Für die Beschäftigten legen die Befunde einen offensiven Umgang mit Fehlern nahe, bei dem Fehler aufgezeigt und thematisiert werden. Die Untersuchungen lieferten keine Hinweise auf Gründe für eine Verschleierung von Fehlern. Der konstruktive Umgang mit den geschilderten Vorfällen deutet darauf hin, dass die Konsequenzen für die Akteure auf Fehleranalyse und Aufklärung zum Zweck zukünftiger Vermeidung ausgerichtet waren. Die Anforderungen des Berufslebens sind geprägt von Unsicherheit und intransparenten Problemen (Gigerenzer, 2002), deren Bewältigung mit einer Strategie der Fehlervermeidung nur unzureichend gelingt (Ulrich, Jick \& Glinow, 1993). Von daher ist ein offensiver Umgang mit Fehlern im Interesse der Beschäftigten, wenn sie ihre Problemlösekompetenz weiter entwickeln wollen.

Lernen aus Fehlern ist also als Voraussetzung für Professional Learning eine Herausforderung für pädagogische Praxis. Es stellen sich aber auch erziehungswissenschaftlichen Forschungsvorhaben Herausforderungen, die möglichen Störquellen solcher Untersuchungen geschuldet sind. So ist zunächst einmal bei einer freiwilligen Teilnahme von einem positiven Bias auszugehen, zumindest von einer Aufgeschlossenheit dem Thema gegenüber. Dies ist bei der Bewertung der Gültigkeit solcher Befunde zu bedenken. Die Untersuchung von Fehlern, dem Umgang mit ihnen und die Analyse von Lernen aus Fehlern werfen darüber hinaus sowohl unter forschungspragmatischen als auch unter forschungsmethodischen Gesichtspunkten Schwierigkeiten auf. Quantitativen Verfahren sind Grenzen gesetzt, weil zum einen - wie die Befunde auch gezeigt haben - kein allgemeingültiges Bewertungsschema für Fehler verfügbar ist und somit erstens nicht klar ist, auf welche Fehlerfälle Versuchspersonen bei der Bearbeitung geschlossener Fragebögen rekurrieren. Zweitens bilden geschlossene Erhebungsinstrumente wie das zur Erfassung der Fehlerorientierung den Grad an Zustimmung zu Generalaussagen ab, die Verzerrungen beispielsweise durch soziale Erwünschtheit unterliegen. Rückschlüsse auf konkretes Verhalten bei Fehlern sind daher problematisch.

Qualitative Verfahren eröffnen die Möglichkeit, konkretes Verhalten in $\mathrm{Zu}-$ sammenhang mit konkreten Fällen abzubilden. Ein Vorteil besteht darin, den Versuchspersonen die Entscheidung über die Überschreitung der Schwelle, ab wann Fehler berichtenswert sind, zu überlassen. Allerdings weisen die vorliegenden Befunde keine Informationen darüber auf, wie repräsentativ die geschilderten Fälle für die tägliche Arbeitspraxis der Versuchspersonen sind. Von daher lässt sich nicht bewerten, ob es sich um Ausnahmefälle oder Alltagssituationen handelt. Solche Informationen wären noch wichtig, um die Gültigkeit der Befunde besser einschätzen zu können.

Aktuelle Publikationen aus der betrieblichen Weiterbildungsforschung zeigen, dass die Schaffung eines Bewusstseins für die Bedeutung des Lernens aus Fehlern im Rahmen der Unternehmenskultur eine der zentralen Herausforde- 
rungen für die Bildungsverantwortlichen in den Betrieben ist (z.B. Arnold \& Lermen, 2004; Faulstich, 2003). Es fällt in ihren Aufgabenbereich, dem Management und der Belegschaft kommunikative Kompetenzen zu vermitteln, die Voraussetzung für eine positive Fehlerkultur sind, bei der Austausch über Vorgehensweisen betrieben und Kritik sowie Verbesserungsvorschläge angebracht werden sollen. Handlungsbedarf ergibt sich aus den Befunden in erster Linie in Hinblick auf die Entwicklung eines gemeinsamen Fehlerverständnisses. Die Moderation eines solchen Prozesses ist möglicherweise die dringendste Aufgabe, die sich aus den Befunden ableiten lässt.

\section{Literatur}

Appelbaum, S. H. \& Gallagher, J. (2000). The competitive advantage of organizational learning. Journal of Workplace Learning, 12, 40-56.

Arnold, R. \& Lermen, M. (2004). Die Systematik des Bedarfs: «Es geht eigentlich um etwas ganz anderes». REPORT - Literatur- und Forschungsreport Weiterbildung, 27 (2), 9-16.

Bauer, J. (2004). Fehlerkultur und epistemische Überzeugungen als Einflussfaktoren individuellen Kompetenzerwerbs am Arbeitsplatz. In H. Gruber, C. Harteis, H. Heid \& B. Meier (Hrsg.), Kapital und Kompetenz - Veränderungen der Arbeitswelt und ihre Auswirkungen aus erziehungswissenschaftlicher Sicht (S. 59-75). Wiesbaden: VS Verlag.

Bauer, J., Festner, D., Harteis, C., Heid, H. \& Gruber, H. (2004). Fehlerorientierung im betrieblichen Arbeitsalltag. Zeitschrift für Berufs- und Wirtschaftspädagogik, 100, 65-82.

Billett, S. (2001). Learning in the workplace. Strategies for effective practice. Crows Nest: Allen \& Unwin.

Cannon, M. D. \& Edmondson, A. C. (2001). Confronting failure: Antecedents and consequences of shared beliefs about failure in organizational work groups. Journal of Organizational Behavior, 22, 161-177.

Dehnbostel, P. (2003). Den Arbeitsplatz als Lernort erschließen und gestalten. GdWZ: Grundlagen der Weiterbildung, 14, 5-9.

Dörner, D. (2003). Die Logik des Misslingens (3. Aufl.). Reinbek: Rowohlt.

Eraut, M. (2002). Developing professional knowledge and competence (7. Aufl.). London: Routledge Falmer.

Faulstich, P. (2003). «Bildungsmanagement» im vernetzten Support. In W. Gieseke (Hrsg.), Institutionelle Innensichten der Weiterbildung (S. 111-128). Bielefeld: Bertelsmann.

Frese, M. \& Zapf, D. (1991). Fehlersystematik und Fehlerentstehung: Ein theoretischer Überblick. In M. Frese \& D. Zapf (Hrsg.), Fehler bei der Arbeit mit dem Computer. Ergebnisse von Beobachtungen und Befragungen im Bürobereich (S. 14-31). Bern: Huber.

Gebauer, A., Groth, T. \& Simon, F. (2004). Aus Fehlern lernen - Scheitern als Chance. Denkanstöße zum lernförderlichen Umgang mit Fehlern und Misserfolgen in Unternehmen. Personalführung, 37 (6), 72-80.

Gigerenzer, G. (2002). Reckoning with risk. Learning to live with uncertainty. London: Penguin Books.

Gruber, H., Harteis, C. \& Rehrl, M. (2005). Professional Learning als Gegenstand internationaler Lehr-Lern-Forschung. Blick in die Wissenschaft, 13 (16), 56-63.

Hacker, W. (1998). Allgemeine Arbeitspsychologie. Bern: Huber.

Harteis, C. (2002). Kompetenzfördernde Arbeitsbedingungen. Zur Konvergenz ökonomischer und pädagogischer Prinzipien betrieblicher Arbeitsorganisation. Wiesbaden: DUV.

Harteis, C., Bauer, J., Festner, D., Gruber, H. \& Heid, H. (2005). Learning from mistakes. Results of an interview-study in German enterprises. Paper präsentiert auf der 86. Jahreskonferenz der American Educational Research Association in Montreal, Kanada. 
Heid, H. (1991). Problematik einer Erziehung zur Verantwortungsbereitschaft. Neue Sammlung, 31, 459-481.

Heid, H. (2005). Übertragung von Verantwortung. In M. Eigenstetter \& M. Hammerl (Hrsg.), Wirtschafts- und Unternehmensethik - ein Widerspruch in sich? (S. 163-182). Kröningen: Asanger.

Hitt, M. A., Black, J. S. \& Porter, L. W. (2005). Management. New Jersey: Pearson.

Hofinger, G. (2002). Erfassung kritischer Zwischenfälle am Beispiel der Luftfahrt und Medizin. In R. Trimpop, B. Zimolong \& A. Kalveram (Hrsg.), Psychologie der Arbeitssicherheit und Gesundheit. Neue Welten - alte Welten (S. 143-148). Heidelberg: Asanger.

Hummel, T. \& Malorny, C. (2002). Total Quality Management. München: Hanser.

Kühl, S. (2000). Das Regenmacher-Phänomen. Widersprüche und Aberglaube im Konzept der lernenden Organisation. Frankfurt/Main: Campus.

Münchrath, R. \& Runge, J. H. (2000). Qualitätsmanagement. In H. Simon (Hrsg.), Das große Handbuch der Strategiekonzepte (S. 82-103). Frankfurt/Main: Campus.

Nierenberg, G. I. (1996). Do it right the first time. A short guide to learning from your most memorable errors, mistakes, and blunders. New York: Wiley.

Norman, D. A. (1989). Dinge des Alltags. Gutes Design und Psychologie für Alltagsgegenstände. Frankfurt/Main: Campus.

Oser, F., Hascher, T. \& Spychiger, M. (1999). Lernen aus Fehlern - zur Psychologie des «negativen Wissens». In W. Althof (Hrsg.), Fehlerwelten - vom Fehlermachen und Lernen aus Fehlern (S. 11-43). Opladen: Leske + Budrich.

Oser, F. \& Spychiger, M. (2005). Lernen ist schmerzhaft. Zur Theorie des Negativen Wissens und zur Praxis der Fehlerkultur. Weinheim: Beltz.

Picot, A., Reichwald, R. \& Wigand, R. T. (2003). Die grenzenlose Unternehmung. Information, Organisation und Management - Lehrbuch zur Unternehmensführung im Informationszeitalter (3. Aufl.). Wiesbaden: Gabler.

Reason, J. T. (1990). Human error. Cambridge: Cambridge University Press.

Reason, J. T. (2001). Understanding adverse events: the human factor. In C. Vincent (Hrsg.), Clinical risk management (S. 9-30). London: BMJ.

Remdisch, S. (2002). Performance improvement through human resource management. In S. Sonnentag (Hrsg.), Psychological management of individual performance (S. 391-402). Chichester: Wiley.

Rybowiak, V., Garst, H., Frese, M. \& Batinic, B. (1999). Error Orientation Questionnaire (EOQ): Reliability, validity, and different language equivalence. Journal of Organizational Behaviour, 20, 527-547.

Simons, P. R.-J. \& Ruijters, M. C. P. (2004). Learning professionals: Toward an intergrated model. In H. P. A. Boshuizen, R. Bromme \& H. Gruber (Hrsg.), Professional learning. Gaps and transitions on the way from novice to expert (S. 207-230). Dordrecht: Kluwer.

Spychiger, M., Oser, F. Hascher, T. \& Mahler, F. (1999). Entwicklung einer Fehlerkultur in der Schule. In W. Althof (Hrsg.), Fehlerwelten. Vom Fehlermachen und Lernen aus Fehlern (S. 43-70). Opladen: Leske + Budrich.

Tjosvold, D., Yu, Z.-Y. \& Hui, C. (2004). Team learning from mistakes: The contribution of cooperative goals and problem solving. Journal of Management Studies, 41, 1223-1245.

Ulrich, D., Jick, T., \& Glinow, M. A. v. (1993). Building and Diffusing Learning Capability. Organizational Dynamics, 22, 52-66

Zapf, D., Frese, M. \& Brodbeck, F. C. (1999). Fehler und Fehlermanagement. In C. Hoyos \& D. Frey (Hrsg.), Arbeits- und Organisationspsychologie (S. 398-411). Weinheim: PVU.

Schlagworte: Instructional Design, Modellentwicklungen, Unterrichtsmodelle, Behaviorismus, Kognitivismus, Konstruktivismus 


\section{Les relations à l'erreur : indicateurs de la culture de l'erreur des entreprises et préalables au développement professionnel}

\section{Résumé}

Les entreprises modernes adoptent une philosophie selon laquelle les employés développent leurs compétences et l'entreprise est considérée comme une organisation apprenante. Ceci est supposé augmenter les capacités de l'entreprise à réagir de manière adéquate à des demandes difficiles à anticiper. Les erreurs constituent dans ce contexte des occasions particulières d'apprendre, d'autant plus que des structures flexibles les rendent plus probables. Cependant, une simple profession de foi en faveur de la culture de l'erreur ne suffit pas pour que l'on en apprenne quelque chose dans le quotidien de l'entreprise. Une relation spécifique à l'erreur est nécessaire, qui ait les caractéristiques d'une culture positive de l'erreur. Cette contribution illustre les conditions d'un apprentissage construit sur les erreurs et donne les résultats de travaux menés avec des employés de diverses entreprises du secteur industriel et de celui des services.

Mots clés: Développement professionnel, culture de l'erreur, entreprise, erreur, culture de l'erreur, entreprise, erreur

\section{La gestione degli errori quale caratteristica della cultura aziendale e premessa per l'apprendimento professionale}

\section{Riassunto}

L'azienda moderna si caratterizza per una filosofia che mira allo sviluppo di competenze e che si concepisce come organizzazione che apprende. Tutto ciò intende migliorare la capacità aziendale di reagire in modo adeguato ad esigenze difficilmente anticipabili. In questo contesto, gli errori costituiscono occasioni di apprendimento speciali, anche perché strutture flessibili tendono maggiormente all'errore. Ma gli atti di fede nei confronti di una cultura dell'errore positiva non sono certo sufficienti per far sì che nella realtà quotidiana dell'azienda si sia effettivamente in grado di apprendere dagli errori. Necessario è uno specifico rapporto con gli errori che risponda a dei requisiti di una cultura dell'errore. Questo contributo chiarisce le condizioni preliminari per un apprendimento dall'errore e discute i risultati di diversi studi condotti con dipendenti di aziende dell'industria e dei servizi.

Parole chiave: apprendimento professionale, cultura dell'errore, azienda, errore 
Handling mistakes in daily working life as an attribute of an inner-firm mistake culture and precondition for professional learning

\section{Summary}

Modern enterprises follow philosophies which require employees to develop competencies and understand their enterprise as a learning organization in order to enhance the potential for appropriate reactions on market changes. Dealing with mistakes is a special source for learning in a scenario where flexible structures increase the probability of their occurrence. A positive way of dealing with mistakes should help to learn from them. However, just a commitment to a positive mistake culture (Fehlerkultur) does not guarantee learning from mistakes in daily working life. This contribution addresses the conditions of learning from mistakes and reports findings from empirical studies with employees.

Keywords: Professional learning, mistake culture, business, mistakes 
T h e m a 